\title{
Tumor protrusion with intensive blood signals on ultrasonography is a strongly suggestive finding of follicular thyroid carcinoma.
}

\author{
Kaoru Kobayashi, Mitsuyoshi Hirokawa, Tomonori Yabuta, Hiroo Masuoka, \\ Mitsuhiro Fukushima, Minoru Kihara, Takuya Higashiyama, Yasuhiro Ito, Akihiro Miya, \\ Nobuyuki Amino, Akira Miyauchi
}

Kuma Hospital, Kobe-City, Hyogo, Japan

\begin{abstract}
Aim: It is difficult to differentiate follicular carcinomas (FC) from follicular adenomas (FA) because ultrasonography and cytology provide very similar findings. FC is histopathologically diagnosed to have capsular and/or vascular invasion. In a few patients, tumor protrusions are observed by preoperative ultrasonography as large forms of capsular invasion of follicular carcinomas. The aim of the study was to clarify the predictive value of tumor protrusions on preoperative ultrasonography for follicular carcinomas. Material and methods: A total of 531 patients (FC: 184 patients, FA: 347 patients) undergoing thyroid surgery were included in this study. A tumor protrusion on ultrasonography was defined as follows: 1) a solid tumor extending beyond a marginal line of capsular zone of the main tumor on B-mode ultrasonography; 2) intensive blood signals observed within the protrusion area on Doppler ultrasonography. The large form of capsular invasion was defined if the protrusion lesion was histopathologically diagnosed to be a capsular invasion of follicular carcinoma. Results: The sensitivity of tumor protrusion was 0.071 , specificity 0.994 , positive predictive value 0.867 , and negative predictive value 0.669 for FC diagnosis. For the large form of capsular invasion in FC the sensitivity of tumor protrusion was 0.813 , specificity 0.982 , positive predictive value 0.684 , and negative predictive value 0.982 . Conclusions: A tumor protrusion with intensive blood signals extending from the main tumor on ultrasonography strongly suggests a large form of capsular invasion of follicular carcinoma.

Keywords: tumor protrusion, thyroid, follicular carcinoma, ultrasonography, capsular invasion
\end{abstract}

\section{Introduction}

Generally, it is impossible to differentiate follicular carcinomas from follicular adenomas by ultrasonic examination and fine-needle aspiration cytology. These tumors are called "follicular neoplasms" or "follicular tumors" after examination by ultrasonography and cytology. Most follicular carcinomas and adenomas demonstrate on ultrasonography a solitary, well-defined, solid, homogeneous, and hypoechoic nodule with a hypoechoic capsular-like zone and no lymph node swelling [1].

Received 20.08.2015 Accepted 25.10.2015

Med Ultrason

2016, Vol. 18, No 1, 25-29

Corresponding author: Kaoru Kobayashi, MD.

8-2-35 Shimoyamate-dori, Chuo-ku,

Kobe-City, Hyogo 650-0011, Japan

Phone:+81-78-371-3721

Fax:+81-78-371-3645

E-mail: kobayashi@kuma-h.or.jp
Follicular carcinomas are known for their propensity to spread by direct infiltration of adjacent thyroid tissue and contiguous venous extension [2,3]. The widely invasive type of follicular carcinoma has a high propensity to invade adjacent thyroid tissue and/ or blood vessels at the macroscopic and microscopic specimens $[2,3]$. We previously described the ultrasonographic features of tumor thrombi showing direct invasion to the veins as a macroscopic or large form of vascular invasion of follicular carcinoma [4]. A macroscopic form of capsular invasion might be detected as a solid mass protruding from the capsule of the main tumor by preoperative ultrasonography in a few patients with the widely invasive type of follicular carcinoma.

We herein report that tumor protrusions on preoperative ultrasonography have a strong predictive value for a large form of capsular invasion of follicular carcinoma. 


\section{Material and methods}

\section{Patients}

We retrospectively reviewed the medical database of patients with follicular carcinoma and follicular adenoma who underwent surgery between January 2007 and December 2012 in our hospital, including the demographic characteristics of patients with tumor protrusions. Preoperative ultrasonography was performed in all patients. The ultrasound readings used in this study were made part of the routine care of these patients by registered sonographers and surgeons. Surgical samples of the thyroid and tumor protrusions were cut before fixation. Specimens were fixed in buffered formalin and embedded in paraffin, and hematoxylin and eosin and immunohistochemical staining was performed. All related pathological specimens were reviewed (by M.H.), and the histopathological diagnosis was included in this study. The preoperative ultrasound readings were verified by surgeons during surgery and by histopathological examination postoperatively. The surgical findings and histopathological diagnosis were recorded in the medical database of the hospital. The ethics committee of our hospital approved the study protocol, which was in adherence to the Declaration of Helsinki.

\section{Ultrasonography}

Preoperative ultrasound examination was performed by well-trained, registered ultrasonographers, using a TOSHIBA Aplio SSA-770A ultrasound system with PLT1204AX (7 - $14 \mathrm{MHz})$ and PLT-805AT (5 - $12 \mathrm{MHz})$ linear probes. In order to make ultrasonic diagnosis of thyroid tumors, our USC system was used (=Ultrasound Class for thyroid tumors at our hospital) [4,5]. All the thyroid tumors were classified into USC 1-5 at ultrasonic diagnosis. Most of follicular carcinomas presented USC3 . We evaluated the intensity of blood signals qualitatively, not quantitatively. We separately evaluated vascularity of thyroid tumors as perinodular and intranodular. Intensity of blood signals was classified into $(-),(+),(++)$, and $(+++)$. We used a standard panel of intensity of blood signals for the reading of the Doppler ultrasonography. Our hospital has 8 or 9 sonographers who were blinded to the laboratory results of the histological diagnosis. The readings of the images before surgery were executed by agreement with two or three sonographers and a surgeon two days before surgery of the patients, as a routine procedure in our hospital. We did not re-evaluate digital images after surgery.

\section{Tumor protrusion and large form of capsular invasion}

A tumor protrusion on ultrasonography was defined as follows: 1) A solid tumor extended beyond a marginal line of capsular zone of the main tumor on B-mode (greyscale) ultrasonography; 2) Intensive blood signals (intranodular intensity; $(++)$ or $(+++))$ within the protrusion lesion. The large form of capsular invasion was defined if the protrusion lesion was histopathologically diagnosed to be a capsular invasion of follicular thyroid carcinoma.

\section{Predictive values of tumor protrusion on ultrasonography}

The relation between tumor protrusion on ultrasonography and histopathological diagnosis was investigated in patients with follicular carcinoma and follicular adenoma, and the predictive values of tumor protrusions on preoperative ultrasonography for histopathological diagnosis were calculated. Then, the relationship between the tumor protrusion on ultrasonography and the large form of the capsular invasion on histopathology was investigated in patients with follicular carcinoma, and the predictive values of tumor protrusions for follicular carcinomas were calculated. We used the statistic software of GraphPad InStat Version 3.0 (GraphPad Software, Inc. La Jolla, CA 92037 USA).

\section{Results}

A total of 531 patients undergoing thyroid surgery were included in this study. There were 184 patients with follicular carcinoma and 347 patients with follicular adenoma. Of these 184 patients with follicular carcinoma, 160 had the minimally invasive type of follicular carcinoma and 24 had the widely invasive type of follicular carcinoma (including 4 patients of oxyphilic cell variant).

Table I shows the relation between the tumor protrusion on ultrasonography and histopathological diagnosis (follicular carcinoma and follicular adenoma). The sensitivity of tumor protrusion on ultrasonography was 0.071 , specificity 0.994 , positive predictive value 0.867 , and negative predictive value 0.669 .

Table II shows the relation between the tumor protrusion on ultrasonography and the large form of capsular invasion on histopathology in follicular carcinoma. The sensitivity of tumor protrusion on ultrasonography was 0.813 , specificity 0.982 , positive predictive value 0.684 , and negative predictive value 0.982 .

Table I. The relation between tumor protrusion on ultrasonography and histopathological diagnosis.

\begin{tabular}{llll}
\hline US findings & FC & FA & Total \\
\hline TP $(+)$ & 13 & 2 & 15 \\
TP $(-)$ & 171 & 345 & 516 \\
Total & 184 & 347 & 531 \\
\hline
\end{tabular}

TP: Tumor protrusion, US: ultrasonography, FC: Follicular carcinoma (184 patients), FA: Follicular adenoma (347 patients). 
Figure 1 shows the ultrasound image, surgical resection material, and histopathological features in patients with a tumor protrusion of follicular carcinoma. The tumor protrusion on ultrasonography was confirmed to be a large form of capsular invasion of follicular carcinoma on histopathology after surgery.

\section{Discussions}

Follicular carcinoma is defined to be a malignant epithelial tumor showing evidence of follicular cell differentiation and lacking the diagnostic nuclear features of papillary carcinoma and is histopathlogically diagnosed to have capsular and/or vascular invasion $[2,3]$. Differential diagnosis of follicular carcinoma from follicular adenoma is preoperatively very difficult, because ultrasonography and cytology provide very similar findings. Classically, follicular carcinomas have been subdivided according to their degree of invasiveness into two major categories [2,3]. Minimally invasive follicular carcinomas have limited capsular and/or vascular invasion, and have an overall good prognosis although some patients may recur and develop metastasis $[7,8]$. In contrast, the widely invasive type of follicular carcinomas have widespread gross invasion of the thyroid parenchyma and

Table II. The relation between tumor protrusion on ultrasonography and the large form of capsular invasion on histopathology in follicular carcinoma.

\begin{tabular}{llll}
\hline US findings & LCI(+) & LCI(-) & Total \\
\hline TP $(+)$ & 13 & 6 & 19 \\
TP $(-)$ & 3 & 162 & 165 \\
Total & 16 & 165 & 184 \\
\hline
\end{tabular}

TP: Tumor protrusion, US: ultrasonography, LCI: Large form of capsular invasion on histopathology; Follicular carcinoma: 184 patients. peri-thyroid soft tissue and/or into blood vessels $[2,3]$ and have a poor prognosis [8].

Most follicular carcinomas and follicular adenomas demonstrate solitary, well-defined, solid, homogeneous, and hypoechoic or isoechoic nodules with a hypoechoic capsular-like zone and no adenopathy on ultrasonography [1]. Therefore, it is considered impossible to differentiate follicular carcinomas from follicular adenomas by ultrasonography. We previously reported a few cases of widely invasive type of follicular carcinoma with tumor thrombus detected by preoperative ultrasonography in combination with cytological findings of "follicular neoplasm". A solid mass in the lumen of veins, that is a tumor thrombus, may be a large form of vascular invasion of widely invasive type of follicular carcinoma [4].

Capsular invasion is histopathologically defined as tumor tissue showing complete transgression of the fibrous capsule, and a tumor bud proliferating beyond the external contour of the capsule $[2,3,9,10]$. In general, capsular invasion is microscopically observed as a mushroom-shaped tumor bud beyond the capsule of the main tumor $[10,11]$. A satellite nodule with the same cellularity and cytoarchitectual features identical to those of the main tumor is regarded as capsular invasion [2,3,11]. Microscopic capsular invasion may develop into a large form in some patients with a widely invasive type of follicular carcinoma. In such a patient, careful ultrasonic examination will provide a typical ultrasound image of the main tumor and tumor protrusions such as a mushroom or protruding navel.

As shown in Table I, tumor protrusion on ultrasonography had a low sensitivity in the histopathological diagnosis of FC and FA due to the fact that tumor protrusion is not a frequent finding in FC. The specificity was high, and this fact reveals that FA does not have tumor protrusion on ultrasonography. The positive and negative predictive values were considerably high revealing

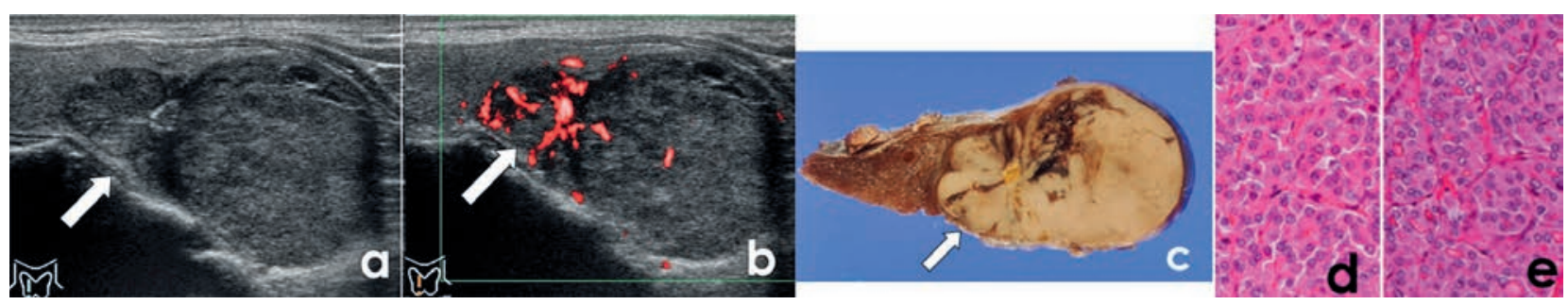

Fig 1. Ultrasound image, surgical resection material, and histopathological features of a patient with a tumor protrusion of follicular carcinoma: a) longitudinal scan of the right thyroid lobe: a solid tumor with tumor protrusion (arrow) in the upper pole. Calcifications are observed between the main tumor and protrusion. The tumor protrusion extends beyond an imaginary line drawn through the outer contour of the capsule; b) Power Doppler ultrasonography: intensive blood signal is present in the tumor protrusion area (arrow); c) Surgical resection material. A solid tumor and a tumor protrusion (arrow) are observed. Tumor tissue of protrusion part projects into the normal thyroid tissue in a mushroom fashion; d) Hematoxylin \& eosin staining in tumor protrusion demonstrating a solid and partly microfollicular growth pattern that consist of oxyphilic cells on histopatholgy; e) Hematoxylin \& eosin staining in main tumor demonstrating identical histopathological features of with tumor protrusion. The diagnosis was follicular carcinoma, a widely invasive type of oxyphilic cell variant, with a large form capsular invasion. 
that the presence or absence of tumor protrusion on ultrasonography expresses histopathological diagnosis to some extent. As shown in Table II, tumor protrusion on ultrasonography presented high sensitivity and specificity for the large form of capsular invasion on histopathology in FC. This fact reveals that the presence or absence of large form of capsular invasion of FC on histopathology is correctly expressed as the presence or absence of tumor protrusion on ultrasonography. The high positive and negative predictive values show that the presence or absence of tumor protrusion on ultrasonography expresses the presence or absence of a large form of capsular invasion on histopathology in FC to a considerably high extent. As mentioned above, the presence of tumor protrusion on ultrasonography is considered to express a large form of capsular invasion of FC.

Some patients were misdiagnosed with tumor protrusions by preoperative ultrasonography. It seems to be difficult to detect tumor protrusions from protrusion-like lesions of irregularly shaped tumors, especially on the dorsal side of the tumors or on the lower side of the tumor near the mediastinum by ultrasonography. High frequency probes (usually $7-14 \mathrm{MHz}$ ) are routinely used for observing thyroid lesions, but it is difficult and they are not suitable for observing deep lesions from the skin. This is the same situation when attempting to detect tumor thrombi of thyroid malignancies by preoperative ultrasonography [4].

Doppler ultrasonography is commonly used to evaluate blood flow through blood vessels, and blood signals are overlaid on the B-mode (grey-scale) ultrasound image. On Doppler ultrasonography, intensive blood signals were shown in the thyroid parenchyma of active Graves' disease [12] or in the tumor area of FC [13-15]. Intensity of blood signals on the solid tissue or tumor detected by Doppler ultrasonography can reflect the functional or proliferative activity of the involved cells. As shown in Figure 1, tumor protrusion areas of FC in the patients demonstrated intensive blood signals on Doppler ultrasonography. Doppler ultrasonography images may reflect the high proliferative activity of tumor cells in the capsular invasion lesion of FC. Modern imaging technology such as real-time elastography is used to evaluate malignant and benign tumors in these days. Some researchers have reported the effectiveness of technology to make a malignant from benign tumors differential diagnosis $[16,17]$. We are now using realtime elastography and shear wave elasticity imaging as well as conventional ultrasonography to distinguish between follicular carcinoma and follicular adenoma, but excellent data are not obtained in our hospital at the present moment.
It is pointless to say that not all follicular carcinomas have tumor protrusions on preoperative ultrasonography. Tumor protrusions as well as tumor thrombi on preoperative ultrasonography, however, have an important predictive value for follicular carcinoma.

The study has some limits. This study was retrospective and ultrasonography and was not performed by the same person. Interobserver and intraobserver agreement was not determined.

\section{Conclusions}

The preoperative detection of a tumor protrusion with intensive blood signals by ultrasonography strongly suggests FC.

Acknowledgement: I would like to express my gratitude to Mr. Hisashi Oota for his technical assistance.

\section{Conflict of interest: none}

\section{References}

1. Wong KT, Ahuja AT. Ultrasound of thyroid cancer. Cancer Imaging 2005; 5: 157-166.

2. Sobrinho Somes M, Noguchi SU. Tumours of the thyroid and parathyroid. Follicular carcinoma. In: DeLellis RA, Lloyd RV, Heitz PU, Eng C (eds). Pathology \& Genetics of Tumours of Endocrine Organs. Lyon, 2004: 67-72.

3. Xu B, Ghossein R. Encapsulated thyroid carcinoma of follicular cell origin. Endocr Pathol 2015; 26: 191-199.

4. Kobayashi K, Hirokawa M, Yabuta T, et al. Tumor thrombus of thyroid Malignancies in veins: Importance of detection by ultrasonography. Thyroid 2011; 21: 527-531.

5. Yokozawa T, Miyauchi A, Kuma K, Sugawara M. Accurate and simple method of diagnosing nodules the modified technique of ultrasound-guided fine needle aspiration biopsy. Thyroid 1995; 5: 141-145.

6. Ito Y, Amino N, Yokozawa T, et al. Ultrasonographic evaluation of thyroid nodules in 900 patients: comparison among ultrasonographic, cytological, and histological findings. Thyroid 2007; 17: 1269-1276.

7. O'Neill CJ, Vaughan L, Learoyd DL, Sidhu SB, Delbridge LW, Sywak MS. Management of follicular thyroid carcinoma should be individualised based on degree of capsular and vascular invasion. Eur J Surg Oncol 2011; 37: $181-185$

8. Ito Y, Hirokawa M, Hiagashiyama T, et al. Prognosis and prognostic factors of follicular carcinoma in Japan: importance of postoperative pathological examination. World J Surg 2007; 31: 1417-1424.

9. Chan JK. Tumors of the thyroid and parathyroid glands. In: Fletcher CD (ed). Diagnostic Histopathology of Tumors. Churchill Livingston, London: 2007: 997-1078. 
10. Rosai J. Follicular carcinoma, In: Rosai J, Carcangiu ML, DeLellis RA Atlas of tumor pathology. Tumors of the thyroid gland. Aemed Forves Institute of Pathology, Washington DC, 1992: 49-63.

11. Ghossein P. Update to the College of American Pathologists Reporting on Thyroid Carcinomas. Head Neck Pathol 2009; 3: 86-93.

12. Ota H, Amino N, Morita S, et al. Quantitative measurement of thyroid blood flow for differentiation of painless thyroiditis from Graves' disease. Clin Endocrinol (Oxf) 2007; 67: 41-45.

13. Fukunari, N, Nagayama M, Sugino K, Mimura T, Ito K, Ito K. Clinical evaluation of color Doppler imaging for the differential diagnosis of thyroid follicular lesions. World J Surg 2004; 28: 1261-1265.
14. Choi YJ, Yun JS, Kim DH. Clinical and ultrasound features of cytology diagnosed follicular neoplasm. Endocr J 2009; 56: 383-389.

15. Iared W, Shigueoka DC, Cristofoli JC, et al. Use of color Doppler ultrasonography for the prediction of malignancy in follicular thyroid neoplasms: systematic review and meta-analysis. J Ultrasound Med 2010; 29: 419-425.

16. Stoian D, Timar B, Derban M, et al. Thyroid Imaging Reporting and Data System (TI-RADS): the impact of Quantitative Strain Elastography for better stratification of cancer risks. Med Ultrason 2015; 17: 327-332.

17. Nell S, Kist JW, Debray TP, et al. Qualitative elastography can replace thyroid nodule fine-needle aspiration in patients with soft thyroid nodules. A systematic review and metaanalysis. Eur J Radiol 2015; 84: 652-661. 